\title{
On the evolutionary status of chemically peculiar stars of the upper main sequence ${ }^{\star}$
}

\author{
H. Pöhnl ${ }^{1}$, H. M. Maitzen ${ }^{1}$, and E. Paunzen ${ }^{1,2}$ \\ 1 Institut für Astronomie der Universität Wien, Türkenschanzstr. 17, 1180 Wien, Austria \\ 2 Zentraler Informatikdienst der Universität Wien, Universitätsstr. 7, 1010 Wien, Austria
}

Received 19 November 2002 / Accepted 28 January 2003

\begin{abstract}
We present further evidence that the magnetic chemically peculiar stars (CP2) of the upper main sequence already occur at very early stages of the stellar evolution, significantly before they reach $30 \%$ of their life-time on the main sequence. This result is especially important for models dealing with dynamo theories, angular momentum loss during the pre- as well as main sequence and evolutionary calculations for CP2 stars. Results from the literature either derived for objects in the Hyades and the UMa cluster or from the Hipparcos mission contradict each other. A way out of this dilemma is to investigate young open clusters with known ages and accurate distances (error $<10 \%$ ), including CP2 members. Up to now, four open clusters fulfill these requirements: IC 2391, IC 2602, NGC 2451 A and NGC 2516. In total, 13 CP2 stars can be found within these clusters. We have used the measurements and calibrations of the Geneva 7-color photometric system to derive effective temperatures and luminosities. Taking into account the overall metallicity of the individual clusters, isochrones and evolutionary tracks were used to estimate ages and masses for the individual objects. The derived ages (between 10 and $140 \mathrm{Myr}$ ) are well in line with those of the corresponding clusters and further strengthen the membership of the investigated CP2 stars.
\end{abstract}

Key words. stars: chemically peculiar - stars: early-type - open clusters and associations: general

\section{Introduction}

A century ago, Antonia Maury (1897) detected a subclass of A-type stars with peculiar lines and line strengths which thereafter became known as Ap (or CP) stars. Subsequently, these stars revealed other peculiar features, e.g. the existence of a strong global magnetic field (Babcock 1947) with a predominant dipole component located at random with respect to the stellar rotation axis and the center of the star as well as overabundances in respect to the Sun for heavy elements such as silicon, chromium, strontium and europium. This lead to the Oblique Rotator concept of slowly rotating stars with noncoincidence of the magnetic and rotational axes (Stibbs 1950).

The peculiar (surface) abundances for CP stars have been explained by the following two theories: 1) Diffusion of chemical elements depending on the balance between gravitational pull and uplift by the radiation field through absorption in spectral lines (Michaud 1970). 2) Selective Accretion from the interstellar medium (Havnes \& Conti 1971) via the stellar magnetic field.

The origin of the magnetic fields is still a matter of debate: those who favor the survival of frozen-in fossil fields

Send offprint requests to: $\mathrm{H}$. M. Maitzen,

e-mail: maitzen@astro.univie.ac.at

^ Based on observations by the Hipparcos satellite. originating from the medium out of which the stars were formed are in opposition to those following the idea that a dynamo mechanism is acting in the interior of these stars.

For many decades the evolutionary status of the CP stars has been controversial. Oetken (1984) concluded that the CP2 (magnetic CP stars) phenomenon appears at the late stages of the main sequence evolution. This theory was further strengthened by the results of Hubrig \& Schwan (1991) and Hubrig \& Mathys (1994) who analysed peculiar objects in the Hyades and the UMa cluster. Hubrig et al. (2000) found that the distribution of CP2 stars of masses below $3 M_{\odot}$ in the HertzsprungRussell-diagram differs from that of the "normal" stars in the same temperature range at a high level of significance: magnetic stars are concentrated toward the center of the main sequence band. In particular, they found that magnetic fields appear only in stars which have already completed at least approximately $30 \%$ of their main sequence life-time. No clear picture emerges as to the possible evolution of the magnetic field across the main sequence. Hints of some (loose) relations between magnetic field strength and other stellar parameters are found: stars with shorter periods tend to have stronger fields, as do higher temperature and higher mass stars. A marginal trend of the magnetic flux to be lower in more slowly rotating stars may possibly be seen as suggesting a dynamo origin for the magnetic field. No correlation between the 
Table 1. Data about our program clusters. The errors in the final digits of the corresponding quantity are given in parenthesis.

\begin{tabular}{lcccc}
\hline \hline Name & NGC 2451 A & NGC 2516 & IC 2391 & IC 2602 \\
& C0743-378 & C0757-607 & C0838-528 & C1041-641 \\
\hline$l / b$ & $252 /-7$ & $274 /-16$ & $270 /-7$ & $290 /-5$ \\
$E(B-V)$ & 0.01 & 0.13 & 0.01 & 0.04 \\
$\pi(\mathrm{Lit})$ & $5.31(19)$ & $2.89(21)$ & $6.85(22)$ & $6.58(16)$ \\
$\pi(\mathrm{our})$ & $5.35(21)$ & $2.69(22)$ & $6.98(18)$ & $6.99(15)$ \\
$d[\mathrm{pc}]$ & $187(7)$ & $372(33)$ & $143(3)$ & $143(3)$ \\
$\log t$ & 7.70 & 8.00 & 7.70 & 7.46 \\
{$[\mathrm{Fe} / \mathrm{H}]$} & -0.26 & -0.23 & -0.04 & -0.20 \\
$n(\mathrm{CP} 2)$ & 1 & 8 & 2 & 2 \\
\hline
\end{tabular}

rotation period and the fraction of the main sequence life time completed is observed, indicating that the slow rotation in these stars must already have been achieved before they became observably magnetic.

The results of the Hipparcos mission on the other hand do not support the mentioned above findings. Gómez et al. (1998) presented the Hertzsprung-Russell-diagram of about $1000 \mathrm{CP}$ stars in the solar neighbourhood using astrometric data from Hipparcos satellite as well as photometric and radial velocity data. Most CP stars lie on the main sequence occupying the whole width of it (about $2 \mathrm{mag}$ ), just like "normal" stars in the same range of spectral types. Their kinematic behaviour is typical of thin disk stars younger than about $1 \mathrm{Gyr}$.

North (1993), North et al. (1997) and Wade (1997) reported that the $\mathrm{CP}$ stars are distributed uniformly along the width of the main sequence with strong magnetic fields existing at all evolutionary states. Stepien (1998) found no evidence that CP2 stars undergo significant angular momentum loss during their main sequence lifetime.

We have investigated CP2 stars of young open clusters in order to tackle the question of whether young peculiar objects exist or not and to put further constraints on theoretical models. Members of open clusters allow to determine precise luminosities and effective temperatures based on reliable calibrations. With the knowledge of accurate distances (e.g. from Hipparcos measurements) and estimates of the overall metallicities, corresponding isochrones can be fitted, resulting in masses and ages for individual $\mathrm{CP} 2$ objects.

We have chosen young open clusters (age $<100 \mathrm{Myr}$ ) including at least one $\mathrm{CP} 2$ with accurate proper motions and distances (error less than 10\%) for our investigation. In total, four open clusters (IC 2391, IC 2602, NGC 2451 A and NGC 2516) fulfill our requirements.

\section{Program clusters}

A main criterion for our target selection was the knowledge of accurate distances. Robichon et al. (1999) have presented parallaxes with errors less than $10 \%$ for nine young open clusters (age $<100 \mathrm{Myr}$ ) of which four have known CP2 members. We have not investigated older open clusters because members of such aggregates have evolved more than $30 \%$ of their main sequence life-time. Such CP2 stars are already known in the Galactic field.
We have slightly modified the values listed in Robichon et al. (1999). They have taken all objects within $10 \mathrm{pc}$ of the individual cluster centers for their determination of the distances. For our work, only objects within three times the cluster radius (taken from Lyngå 1987) were considered. Furthermore, we have discarded objects with proper motions deviating more than $0.221 \pi$ [mas yr $\left.{ }^{-1}\right]\left(=1 \mathrm{~km} \mathrm{~s}^{-1}\right)$ from the calculated overall proper motion of the individual open cluster. Table 1 lists these parallaxes with those of Robichon et al. (1999) and other characteristics of our program clusters. All parallaxes agree very well and are also consistent with results from van Leeuwen (1999).

\subsection{IC 2391}

This young open cluster contains two CP2 stars according to North (1984) and Maitzen \& Catalano (1986). Both objects are members of IC 2391 (Perry \& Hill 1969; numbering system).

- HD 74169 (\#18): classified as Ap EuCr(Sr) by Buscombe (1965), Houk (1978) and Levato \& Malaroda (1984).

- HD 74535 (\#31): peculiar according to the same references as listed above.

The values for the metallicity and reddening were taken from Lyngå (1987).

\subsection{IC 2602}

The overall characteristics of this open cluster was only investigated by Lyngå (1987) who lists $\log t=7.46, E(B-V)=0.04$ and $[\mathrm{Fe} / \mathrm{H}]=-0.20$. The numbering in brackets is according to Braes (1962).

- HD 92385 (\#71): Bidelman \& McConnell (1973) and Houk \& Cowley (1975) establish the membership and the peculiar nature which was confirmed by Maitzen et al. (1988).

- HD 92664 (\#27): additional classification as B9p (Si) by Whiteoak (1961) and Abt \& Morgan (1972).

The $\Delta a$ measurements of Maitzen et al. (1988) for both objects show only moderate peculiarity.

\subsection{NGC 2451 A}

What was believed to be one open cluster was actually separated into one less and one more distant aggregate within 
one line of sight (Eggen 1983; Maitzen \& Catalano 1986). Röser \& Bastian (1994) postulated two clusters at distances of 220 and $400 \mathrm{pc}$ which was later confirmed by Platais et al. (1996). Carrier et al. (1999) used the Hipparcos data and found two similar aged clusters $(\log t=7.70)$ at distances of 197 (NGC 2451 A) and 358 pc (NGC 2451 B). For NGC 2451 A they list 28 members including the peculiar object HD 63401 (\#277; Williams 1967). This object was classified as Ap Si by Bidelman \& McConnell (1973) and Houk (1982) whereas Hartoog (1976) lists B8III. The photometric CP2 star HD 62992 (\#250) is a non-member according to Carrier et al. (1999).

\subsection{NGC 2516}

This open cluster contains at least eight CP2 stars for which spectral classification resolution spectroscopy as well as $\Delta a$ photometry is available (Maitzen \& Hensberge 1981). Mermilliod (1981) gives an age $\log t=8.03$ whereas Lyngå (1987) lists a value of 7.85. Jeffries et al. (1997) derived a metallicity of $[\mathrm{Fe} / \mathrm{H}]=-0.32(6)$ for cooler members. Additional estimates were made by Cameron $(1985 ;-0.42)$, Lyngå (1987; -0.23) and Debernardi \& North (2001; 0). The metallicity calibration of Eggen (1972) for the Johnson $U B V$ system results in a value of -0.23 whereas Strömgren $u v b y$ photometry gives -0.20 (Snowden 1975; Berthet 1990). Taking all these values into account, we have chosen to use the value of Lyngå (1987) as a "mean" metallicity: $[\mathrm{Fe} / \mathrm{H}]=-0.23$.

Maitzen \& Hensberge (1981) have reported a significant differential reddening throughout the cluster area. We have used the $Q$-method (Johnson \& Morgan 1953) to calculate the overall reddening as well as the individual values for the CP2 stars. The derived values are well in agreement with the results listed by Lyngå (1987).

The following stars are probably CP2 stars (the numbering is according to Cox 1955):

- HD 65712 (not listed in Cox 1955): Dachs \& Kabus (1989) list a spectral type of A0p (SiII) and a high membership probability consistent with the results of Bidelman \& McConnell (1973). Maitzen \& Hensberge (1981) measured a very high $\Delta a$-value of +70 mmag.

- HD 65987 (\#15): A possible binary system (Abt \& Levy 1972; Snowden 1975; Gieseking 1978) classified as B9.5IVp (SiII) and B9p Si by Abt \& Morgan (1969) and Hartoog (1976), respectively.

- HD 66295 (\#26): B8/9IV Si according to Houk \& Cowley (1975), listed as member in Dachs \& Kabus (1989).

- HD 66318 (\#24): member, classified as A0pEuCrSr (Hartoog 1976).

- CP-60 944A (\#208) and B (\#209): the objects have spectral types of B8 III and B8p Si with an apparent confusion about the identification of the individual components.

- CP-60978 (\#c): a member (Dachs \& Kabus 1989) classified as A0p (SiII) and A0p EuCrSr (Abt \& Morgan 1969; Hartoog 1976).
- CP-60981 (\#38): visual binary with a period of $3.2 \mathrm{~d}$ (North 1984) with a classification of $\mathrm{A} 2 \mathrm{Vp}(\mathrm{SrCrEu})$ given by Hartoog (1976).

- CP-60948 (\#8), CP-60 1008 (\#17) and CP-60 1013(\#28): Maitzen \& Hensberge (1981) measured $\Delta a$ of +27 (\#17) and +13 mmag (\#8 and \#28); no other data in the literature were found.

The apparent peculiarity of CP-60948 (\#8), CP-601008 (\#17) and CP-601013(\#28) should be confirmed by further spectroscopic data.

New observations at the VLT (Wade, private communication) revealed a very strong magnetic field $(4.5 \mathrm{kG}$ longitudinal field, $15 \mathrm{kG}$ surface field) in HD 66318 . Weaker fields were found for HD 66295 ( $-0.8 \mathrm{kG}$ longitudinal) and CP-60 944A $(0.6 \mathrm{kG})$. No magnetic fields were detected for CP-60948, $\mathrm{CP}-60981$ or any other above listed objects. These results are in excellent agreement with the measurements within the photometric $\Delta a$-system.

\section{Determination of the evolutionary status for the CP2 stars}

For the further analysis of the evolutionary status of the $\mathrm{CP} 2$ objects, the program stars were located in a $\log L / L_{\odot}$ versus $\log T_{\text {eff }}$ diagram to derive masses and ages. In the following subsections we will discuss this procedure in more detail (see also Pöhnl 2001).

\subsection{Effective temperature}

Since all of our program stars were measured within the Geneva 7-color photometric system (Rufener 1988) we have taken the relevant calibrations within this system. The most recent calibration for the effective temperature in the range from 5800 to $15000 \mathrm{~K}$ is given by Künzli et al. (1997). However, this calibration has to be treated with caution for CP2 stars because these objects show a "blueing" effect which manifests in hotter calibrated temperatures due to stronger UV absorption than in normal type stars (Adelman 1980; Maitzen 1980). We have therefore used the modifications for peculiar objects as given by Hauck \& North (1993) and Hauck \& Künzli (1996). For the calibration within the temperatures an a-priori knowledge of the metallicity and reddening is necessary (although the Geneva $X$ and $Y$ indices are unaffected by reddening). The small reddening (Table 1) of our program clusters has been accounted for. The metallicities listed in the literature for IC 2602, NGC 2451 A and NGC 2516 were taken into account since they deviate significantly from solar values.

Hubrig et al. (2000) have compared the results of the effective temperature calibration from the Geneva 7-color photometric system with those of spectroscopic investigations as well as the infrared flux method and find a satisfying agreement within the range between 7500 and $15000 \mathrm{~K}$.

The accuracy of the photometric calibration for normal type stars is given between \pm 62 and $\pm 386 \mathrm{~K}$ depending on the temperature range (Hauck \& Künzli 1996). Such error values are statistically derived for a large sample of objects and have to 
Table 2. Summary of results for true CP2 stars in our program clusters; HD 65987 and CP-60981 are binary systems for which we list the values for the primary component. The errors in the final digits of the corresponding quantity are given in parenthesis; $\tau$ is the age of the star, $\tau_{\mathrm{HR}}$ the time for a star on the main sequence and $\tau_{\mathrm{Cl}}$ the known age of the individual cluster.

\begin{tabular}{lcccccccc}
\hline \hline Cluster & $\begin{array}{c}{[\mathrm{Fe} / \mathrm{H}]} \\
{[\mathrm{dex}]}\end{array}$ & Object & $\log T_{\text {eff }}$ & $\log L / L_{\odot}$ & $M / M_{\odot}$ & $\begin{array}{c}\tau \\
{[\mathrm{Myr}]}\end{array}$ & $\begin{array}{c}\tau_{\mathrm{HR}} \\
{[\mathrm{Myr}]}\end{array}$ & $\tau_{\mathrm{Cl}} / \tau_{\mathrm{HR}}$ \\
\hline NGC 2451 A & -0.26 & HD 63041 & $4.155(19)$ & $2.26(8)$ & $3.58(11)$ & 20 & 215 & 0.23 \\
NGC 2516 & -0.23 & HD 65712 & $4.002(19)$ & $1.42(12)$ & $2.16(9)$ & 90 & 870 & 0.12 \\
& & HD 65987 & $4.107(19)$ & $2.31(16)$ & {$[3.18]$} & {$[105]$} & {$[295]$} & {$[0.36]^{1}$} \\
& & HD 66295 & $4.039(19)$ & $1.64(12)$ & $2.44(10)$ & 95 & 615 & 0.17 \\
& & HD 66318 & $3.979(15)$ & $1.29(81)$ & $2.00(5)$ & 140 & 1100 & 0.10 \\
& & CP-60 944A & $4.104(19)$ & $2.10(12)$ & $3.14(13)$ & 105 & 305 & 0.33 \\
& & CP-60 944B & $4.096(19)$ & $1.96(12)$ & $3.03(12)$ & 105 & 340 & 0.32 \\
IC 2391 & & CP-60978 & $4.076(19)$ & $1.90(12)$ & $2.82(7)$ & 110 & 410 & 0.26 \\
& & CP-60 981 & $3.975(15)$ & $1.51(8)$ & {$[1.95]$} & {$[100]$} & {$[1180]$} & {$[0.10]^{2}$} \\
IC 2602 & -0.04 & HD 74169 & $3.998(19)$ & $1.41(7)$ & $2.25(10)$ & $<10$ & 775 & 0.07 \\
& & HD 74535 & $4.418(19)$ & $2.33(7)$ & $3.80(11)$ & 35 & 185 & 0.29 \\
& -0.20 & HD 92385 & $4.043(19)$ & $1.69(6)$ & $2.47(6)$ & 95 & 590 & 0.05 \\
& & HD 92664 & $4.176(19)$ & $2.47(6)$ & $3.99(12)$ & 45 & 165 & 0.18 \\
\hline
\end{tabular}

${ }^{1}$ Probable binary; ${ }^{2}$ Binary.

be treated as such. North (1998) investigated 46 CP2 stars and found an error of the mean of $4.4 \%$ and $3.4 \%$ for objects hotter and cooler than $9500 \mathrm{~K}$, respectively. These percentages transform into $\Delta \log T_{\text {eff }}=0.019$ and 0.014 which seems reasonable also for our error estimation.

\subsection{Luminosity}

The luminosity calibration for CP2 stars is also affected by stronger UV absorption. We have followed the approaches presented by Lanz (1984), Stepien (1994) and North (1998) who list correction factors $\delta_{B C}$ for the estimation of bolometric magnitudes for chemically peculiar stars. The values for $\delta_{B C}$ reach up to $0.3 \mathrm{mag}$ for objects in the range $-0.85<(B 2-G)_{0}<-0.67$ within the Geneva 7-color photometric system. The luminosity can be calculated as:

$\log L / L_{\odot}=0.4 \times\left(4.72-M_{V}-B C+\delta_{B C}\right)$

with

$M_{V}=m_{V}+5 \times \log \pi+5-3.1 \times E(B-V)$.

The values for the bolometric correction $B C$ and the standard solar value were taken from Flower (1996).

We have also corrected for the so-called "Lutz-Kelker effect". Lutz \& Kelker (1973) were among the first to calculate corrections for the bias in the absolute magnitude of a star as estimated from its trigonometric parallax. The bias is introduced by ubiquitous random errors of measurements which, on average, cause the trigonometric parallax to be overestimated. The correction for our program clusters range between 0.004 and $0.028 \mathrm{mag}$.

The error of the luminosity based on the relative error of the parallax is given as:

$\Delta \log L / L_{\odot}=2 \times \log \left(1+\frac{\sigma(\pi)}{\pi}\right)$.

The errors of our calibrations (Table 2) are compatible with those listed by e.g. North (1998).

\subsection{Age and mass}

As a next step, we have used the main sequence evolutionary tracks and isochrones from Schaller et al. (1992), Charbonnel et al. (1993) and Schaerer et al. (1993a, b) in order to estimate the individual masses and ages for our program stars. We have compared these models to those given by Claret \& Gimenez (1992) and Bressan (1993) and found no significant deviations between them (see also Lastennet et al. 1999 for such a comparison).

The models used are available for different metallicities and masses. For the determination of the masses and ages we have used the grids as tabulated with the hydrogen concentration in the core $X_{\mathrm{C}}, \log L / L_{\odot}$ and $\log T_{\text {eff }}$.

Our analysis shows that, for example, the values of $\log L / L_{\odot}$ at the zero age main sequence are nearly linear correlated with the values for $\log M / M_{\odot}$. The same correlation exists for the values at the terminal age main sequence. In addition we find that the $\log L / L_{\odot}$ and $\log M / M_{\odot}$ values are, at any stage of the stellar evolution, well correlated with $X_{\mathrm{C}}$. Since the data of the used model show that there is a clear dependance of the time for a star on the main sequence $\tau_{\mathrm{HR}}$ and $\log M / M_{\odot}$ which can be written as

$\log \tau_{\mathrm{HR}}=9.99-3.36 \times \log \left(M / M_{\odot}\right)+0.67 \times \log ^{2}\left(M / M_{\odot}\right)$

we can use the result of this quadratic regression for the relative age $\tau / \tau_{\mathrm{HR}}$. This parameter is independent of the stellar masses and can be described via $X_{\mathrm{C}}$ as

$\tau / \tau_{\mathrm{HR}}=1.004-0.331 \times X_{\mathrm{C}}-1.653 \times X_{\mathrm{C}}^{2}$.

The error for the relative age is less than $\pm 5 \%$. Finally, we have calculated the star's ages via the above given equations. The grid between the zero and terminal age main sequence is defined by lines of equivalent $M$ and $X_{\mathrm{C}}$. With known values for $\log L / L_{\odot}$ and $\log T_{\text {eff }}$, a box in which we have interpolated can be determined. This quadratic interpolation results in $M$ as well as $X_{\mathrm{C}}$ and thus the age of the individual program stars. 


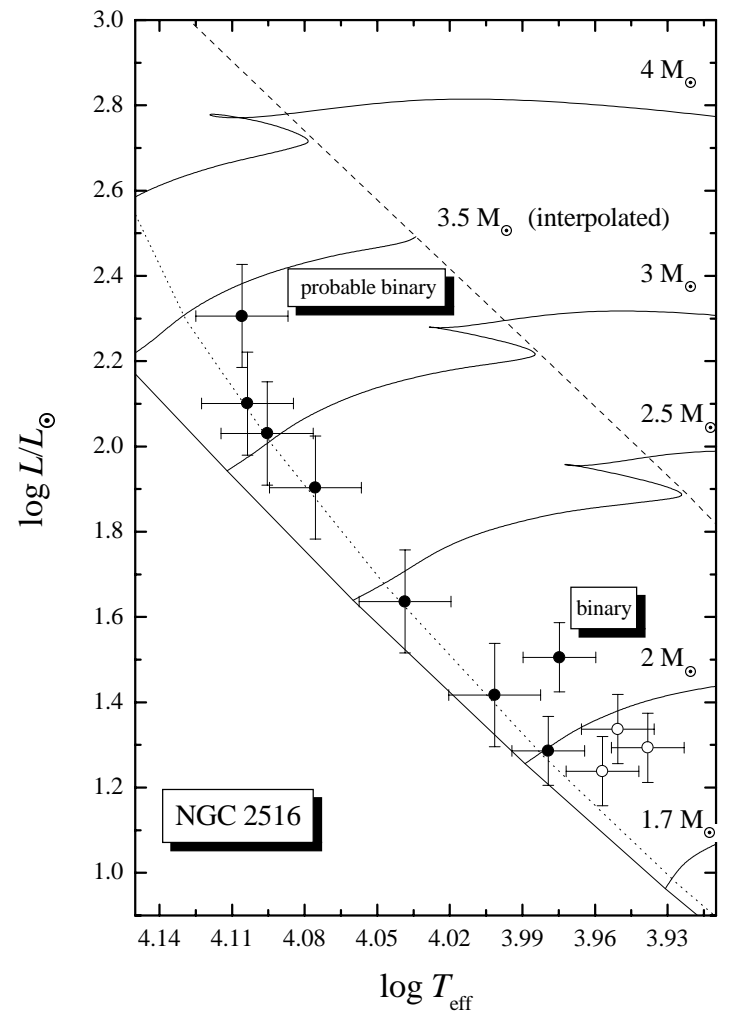

Fig. 1. The location of the CP2 stars for NGC 2516 taken from Table 2. Filled circles are well established CP2 objects and open circles are doubtful cases (CP-60 948, CP-60 1008 and $\mathrm{CP}-60$ 1013). The dotted line is the isochrone for $\log t=8.00$ and $[\mathrm{Fe} / \mathrm{H}]=-0.23$ taken from Schaller et al. (1992) whereas the dashed line denoted the terminal age main sequence; the evolutionary tracks for individual masses are interpolated within the ones listed by Schaller et al. (1992), Schaerer et al. (1993a,b) and Charbonnel et al. (1993).

\section{Results}

All results for the CP2 stars in the four open clusters are summarized in Table 2. In the following we will discuss the objects in the open clusters in more detail.

IC 2391, IC 2602 and NGC 2451 A: All CP2 stars are members of the individual clusters with very young ages.

$N G C$ 2516: Figure 1 shows the location of the CP2 stars together with the isochrone for $\log t=8.00$ and $[\mathrm{Fe} / \mathrm{H}]=-0.23$ and the evolutionary tracks for individual masses. All but two (HD 65987 and CP-60 981) lie very well on the apparent main sequence of this cluster and are members of it. The two deviating objects are both binaries. Their location above the corresponding isochrone can be very well reproduced taking the binarity into account. The masses and ages for the visual binary system CP-60 944A + B (both in very good agreement with the values from Debernardi \& North 2001) infer that they are physically coupled. The mean value of the age for all six single CP2 stars is 106(17) Myr which is in excellent agreement with the overall age of this cluster (100 Myr).

The location of all investigated CP2 stars within the relevant Hertzsprung-Russell-diagrams qualify them as being members of the corresponding aggregate. Furthermore, their ages $(10<$ ages $<140 \mathrm{Myr})$ are within the expected error of the overall age of the individual open cluster. This proves that CP2 stars do exist at very young evolutionary stages, clearly before they have reached $30 \%$ of their main sequence life-time.

\section{Conclusions}

Several publications during the last decades have been dedicated to the evolutionary status of the CP2 stars. Still this issue is a matter of debate. In principle, there are two working hypotheses: 1) CP2 stars show their peculiar nature soon after arriving at the zero age main sequence or 2) after about $30 \%$ of their life-time on the main sequence, the phenomenon occurs.

We have investigated four young (age not more than $100 \mathrm{Myr}$ ) open clusters with known CP2 members. The peculiarity of these objects was established via photometric as well as spectroscopic data. The program clusters were chosen on the basis of available Geneva 7-color photometry and known accurate Hipparcos distances (error $<10 \%$ ).

We have derived effective temperatures and luminosities for these objects and calibrated ages as well as masses with the help of standard evolutionary models taking the overall metallicities of the individual clusters into account.

All investigated objects are members of their open cluster with ages between 10 and $140 \mathrm{Myr}$ corresponding to a relative age of 0.05 and 0.36 of their main sequence life-time. This clearly proves that the observable $\mathrm{CP} 2$ phenomenon occurs already well before a star has reached $30 \%$ of its life-time on the main sequence.

The somewhat discrepant result of Hubrig et al. (2000) can be understood by their bias in selecting objects (not by differences in the reduction procedure): the detection of resolved Zeeman patterns requires a specifically slow rotation. This gives preference to finding such objects in advanced phases on the main sequence band where rotational velocities have been decreased by the growth of stellar radii.

Concerning the question of a parallel evolution of both the magnetic and spectroscopic peculiarities, it seems still premature to draw conclusions from the existing data. A concentrated effort to observe both essential features of the magnetic peculiar stars is highly desirable in order to reveal their nature.

Acknowledgements. Use was made of the SIMBAD database, operated at CDS, Strasbourg, France. EP acknowledges partial support from the Fonds zur Förderung der wissenschaftlichen Forschung, project P14984.

\section{References}

Abt, H. A., \& Levy, S. G. 1972, ApJ, 172, 355

Abt, H. A., \& Morgan, W. W. 1969, AJ, 74, 813

Abt, H. A., \& Morgan, W. W. 1972, ApJ, 174, L131

Adelman, S. J. 1980, A\&A, 89, 149

Babcock, H. W. 1947, ApJ, 105, 105

Berthet, S. 1990, A\&A, 236, 440

Bidelman, W. P., \& McConnell, D. J. 1973, AJ, 78, 687

Braes, L. L. E. 1962, Bull. Astron. Inst. Netherl., 16, 297

Bressan, A., Fagotto, F., Bertelli, G., \& Chiosi, C. 1993, A\&AS, 100, 647 
Buscombe, W. 1965, MNRAS, 129, 411

Cameron, L. M. 1985, A\&A, 146, 59

Carrier, F., Burki, G., \& Richard, C. 1999, A\&A, 341, 469

Charbonnel, C., Meynet, G., Maeder, A., Schaller, G., \& Schaerer, D. 1993, A\&AS, 101, 415

Claret, A., \& Gimenez, A. 1992, A\&AS, 96, 255

Cox, A. N. 1955, ApJ, 119, 188

Dachs, J., \& Kabus, H. 1989, A\&AS, 78, 25

Debernardi, Y., \& North, P. 2001, A\&A, 374, 204

Eggen, O. J. 1972, ApJ, 173, 63

Eggen, O. J. 1983, AJ, 88, 197

Flower, P. J. 1996, ApJ, 469, 355

Gieseking, F. 1978, A\&AS, 32

Gómez, A. E., Luri, X, Grenier, S., et al. 1998, A\&A, 336, 953

Hartoog, M. R. 1976, ApJ, 205, 807

Hauck, B., \& Künzli, M. 1996, Baltic Astron., 5, 303

Hauck, B., \& North, P. 1993, A\&A, 269, 403

Havnes, O., \& Conti, P. S. 1971, A\&A, 14, 1

Houk, N. 1978, University of Michigan Catalogue of Pöhnl, H. 2001, Ph.D. Thesis, University of Vienna

Two-Dimensional Spectral Types for the HD stars, vol. II, Robichon, N., Arenou, F., Mermilliod, J.-C., \& Turon, C. 1999, A\&A, Astronomy Dept., Univ. Mich., Ann Arbor, Michigan

Houk, N. 1982, University of Michigan Catalogue of Two-Dimensional Spectral Types for the HD stars, vol. III, Astronomy Dept., Univ. Mich., Ann Arbor, Michigan

Houk, N., \& Cowley, A. P. 1975, University of Michigan Catalogue of Two-Dimensional Spectral Types for the HD stars, vol. I, Astronomy Dept., Univ. Mich., Ann Arbor, Michigan

Hubrig, S., \& Mathys, G. 1994, AN, 5, 343

Hubrig, S., North, P., \& Mathys, G. 2000, A\&A, 539, 352

Hubrig, S., \& Schwan, H. 1991, A\&A, 251, 469

Jeffries, R. D., Thurston, M. R., \& Pye, J. P. 1997, MNRAS, 287, 350

Johnson, H. L., \& Morgan, W. W. 1953, ApJ, 117, 313

Künzli, M., North, P., Kurucz, R. L., \& Nicolet, B. 1997, A\&AS, 122, 51

Lanz, T. 1984, A\&A, 139, 161

Lastennet, E., Valls-Gabaud, D., Lejeune, Th., \& Oblak, E. 1999, A\&A, 349, 485

Levato, H., \& Malaroda, S. 1984, Astrophys. Lett., 24, 37

Lutz, Th. E., \& Kelker, D. H. 1973, PASP, 85, 573

Lyngå, G. 1987, Catalogue of Open Cluster Data, 5th edition, CDS, Strasbourg
Maitzen, H. M. 1980, A\&A, 89, 230

Maitzen, H. M., \& Catalano, F. A. 1986, A\&AS, 66, 37

Maitzen, H. M., \& Hensberge, H. 1981, A\&A, 96, 151

Maitzen, H. M., Schneider, H., \& Weiss, W. W. 1988, A\&AS, 75, 391

Maury, A. 1897, Ann. Astron. Obs. Harvard, vol. 28, Part 1

Mermilliod, J.-C. 1981, A\&A, 97, 235

Michaud, G. 1970, ApJ, 160, 641

North, P. 1984, A\&AS, 55, 259

North, P. 1993, in Peculiar versus Normal Phenomena in A-type and Related Stars, ed. M. M. Dworetsky, R. Castelli, \& R. Faraggiana, ASP Conf. Ser., 44, 577

North, P. 1998, A\&A, 334, 181

North, P., Jaschek, C., Hauck, B., et al. 1997, Proc. of the ESA Symp. Hipparcos Venice 97, ESA SP-402, 239

Oetken, L. 1984, AN, 306, 187

Perry, C. L., \& Hill, G. 1969, AJ, 74, 899

Platais, I., Kozhurina-Platais, V., Barnes, S., \& Horch, E. P. 1996, BAAS, 28, 822 345,471

Röser, S., \& Bastian, U. 1994, A\&A, 285, 875

Rufener, F. 1988, Catalogue of Stars measured in the Geneva Observatory Photometric System (4th ed.), Observatoire Geneve

Schaerer, D., Meynet, G., Maeder, A., \& Schaller, G. 1993a, A\&AS, 98,523

Schaerer, D., Charbonnel, C., Meynet G., Maeder, A., \& Schaller, G. 1993b, A\&AS, 102, 339

Schaller, G., Schaerer, G., Meynet, G., \& Maeder, A. 1992, A\&AS, 96, 269

Snowden, M. S. 1975, PASP, 87, 721

Stepien, K. 1994, in Chemically Peculiar \& Magnetic Stars, ed. J. Zwerko, \& J. Ziznovsky, 8

Stepien, K. 1998, Contributions of the Astronomical Observatory Skalnaté Pleso, vol. 27, 205

Stibbs, D. W. N. 1950, MNRAS, 110, 395

van Leeuwen, F. 1999, A\&A, 341, L71

Wade, G. A. 1997, A\&A, 325, 1063

Whiteoak, J. B. 1961, MNRAS, 123, 245

Williams, P. M. 1967, Mon. Not. R. Astron. Soc. Afr., 25, 122 